\title{
SOROVARES DE LEPTOSPIRAS PREDOMINANTES EM EXAMES SOROLÓGICOS DE BUBALINOS, OVINOS, CAPRINOS, EQÜINOS, SUÍNOS E CÃES DE DIVERSOS ESTADOS BRASILEIROS
}

\author{
MOST FREQUENT SEROVARS OF LEPTOSPIRES IN SEROLOGICAL \\ TESTS OF BUFFALOES, SHEEPS, GOATS, HORSES, SWINES \\ AND DOGS FROM SEVERAL BRAZILIAN STATES
}

\author{
Andrea Cecília Mercaldi Favero ${ }^{1}$ Sônia Regina Pinheiro ${ }^{2}$ Silvio Arruda Vasconcellos ${ }^{3}$ \\ Zenáide Maria Morais ${ }^{4}$ Fernando Ferreira $^{5}$ José Soares Ferreira Neto $^{6}$
}

RESUMO

Em estudo retrospectivo abrangendo os anos de 1984 a 1997, foram realizados 15.558 exames sorológicos para leptospirose (SAM, com coleção de 24 sorovares), que incluíram: 284 ovinos, 879 bubalinos, 983 cães, 1.941 caprinos, 2.903 eqüinos e 8.568 suínos, distribuídos percentualmente por estado da seguinte forma: ovinos - SP (100\%); bubalinos - SP (100\%); cães - SP (80,7\%), RS (0,10\%), SC (0,10\%) e PI $(19,0 \%)$; caprinos - SP $(33,1 \%), P B(63,7 \%)$ e $C E(3,2 \%)$; eqüinos $-S P$ (79,3\%), RS (9,98\%), SC (0,62\%), PR (2,5\%), RJ (0,17\%), MG (1,96\%), MT (3,99\%), PB (1,3\%) e PI (0,03\%); suinos - SP (61,91\%), RS (0,3\%), SC (5,95\%), PR (3,67\%), RJ (0,88\%), MG (24,38\%), GO (1,12\%), SE (0,2\%), PE (0,90\%), CE (0,34\%) e MA $(0,1 \%)$. A distribuição temporal dos animais examinados incluiu: para a espécie ovina - 54,5\% referentes aos anos $1996 e$ $97,33,3 \%$ referentes a 89 e 90 e 12,2\% aos outros anos; espécie bubalina - 21,7\% no intervalo de 1984 a 95 e 78,83\% entre 96 e 97; espécie canina - 16,91\% entre 1984 e 92 e 83,09\% entre 1993 e 97; caprinos - 6,97\% entre 1984 e 91 e 93,09\% entre 1992 e 97, sendo que 49\% das amostras foram referentes ao ano de 1992; eqüinos - 18,1\% no intervalo de 84 a 90 e $81,9 \%$ de1991 a 97; suinos - 61,16\% referentes aos anos 90, 91, $95 e$ 96. As médias de animais reatores e variantes mais freqüentes por espécie foram: ovinos $-0,70 \%$ de soropositividade e reações mais freqüentes para a variante icterohaemorrhagiae; bubalinos - 43,7\% de positivos e variantes hardjo seguida de pomona; cães - soropositividade de $17,7 \%$ e reaçóes para as variantes copenhageni e icterohaemorrhagiae no estado de SP e pyrogenes no PI; caprinos - $4,17 \%$ de positividade e variantes icterohaemorrhagiae e grippotyphosa no CE, icterohaemorrhagiae na $P B$ e pyrogenes em $S P$; eqüinos - $29 \%$ de soros positivos e variantes icterohaemorrhagiae no $P R, S C$, $S P, R J$ e $M G$, grippotyphosa no $M T$, pyrogenes na $P B$ e patoc no $R S$; suinos - soropositividade de $24,46 \%$ e grippotyphosa seguida de icterohaemorrhagiae em $M G$, pomona no $R S$, pomona $e$ icterohaemorrhagiae em $P E$ e RJ, autumnalis no $C E$ e icterohaemorrhagiae em GO, PR, SC e SP.

Palavras-chave: sorologia, aglutinação, leptospirose, bubalinos, ovinos, caprinos, eqüinos, suínos, cães.

\section{SUMMARY}

From 1984 to 199715.558 sorologic tests to leptospirosis were performed (MAT with 24 leptospires serovars) in 284 sheeps, 879 boffaloes, 983 dogs, 1.941 goats, 2.903 horses and 8.568 swines. The distribuition of animals examined by species and state were: sheeps - SP (100\%); buffaloes - SP (100\%); dogs - SP (80.7\%), RS (0.10\%), SC (0.10\%) and PI (19.0\%); goats - SP (33.1\%), $P B(63.7 \%)$ and $C E(3.2 \%)$; horses - SP (79.3\%), RS (9.98\%), SC (0.62\%), PR (2.5\%), RJ (0.17\%), $M G(1.96 \%), M T(3.99 \%), P B(1.3 \%)$ and PI $(0.03 \%)$; swines $S P(61.91 \%), R S(0.3 \%), S C(5.95 \%), P R(3.67 \%), R J(0.88 \%)$, $M G(24.38 \%), G O(1.12 \%), S E(0.2 \%), P E(0.90 \%), C E(0.34 \%)$ and MA (0.1\%). From the sheeps tested, $54.5 \%$ were examined between 1996 and $97.33 .3 \%$ between 1989 and 1990 and $12.2 \%$ in the other years; buffaloes - 21.7\% from 1984 to 95 and 78.83\% between 1996 and 97; dogs-16.91\% from 1984 to 92 and $83.09 \%$ from 1993 to 97; goats - 6.97\% from 1984 to 91 and 93.09\% from 1992 to 97, althought 49\% were concerning to 1992; horses - 18.1\% from 84 to 90 and $81.9 \%$ from 1991 to 97; swines - 61.16\% concerning 1990, 91, 95 and 96. The proportion

\footnotetext{
${ }^{1}$ Médico Veterinário, Pós-graduanda da Faculdade de Medicina Veterinária e Zootecnia(FMVZ) da Universidade de São Paulo (USP).

${ }^{2}$ Médico Veterinário, Professora Doutora da FMVZ, USP, Av, Prof. Dr. Orlando Marques de Paiva 87, 05508-000 São Paulo, SP. Email: soniapin@usp.br

${ }^{3}$ Médico Veterinário, Professor Titular da FMVZ, USP.

${ }^{4}$ Bióloga, Técnica Especializada de Nível Superior da FMVZ, USP.

${ }^{5}$ Médico Veterinário, Professor Doutor da FMVZ, USP .

${ }^{6}$ Médico Veterinário, Professor Associado da FMVZ, USP.
} 
of reactors to at least one leptospira serovar presented the following average and most frequent serovars: sheeps $-0.70 \%$ and icterohaemorrhagiae like most frequent serovar; buffaloes $43.7 \%$ and serovars hardjo and pomona; $\operatorname{dogs}-17.7 \%$ and serovar icterohaemorrhagiae in SP and pyrogenes in PI; goats $4.17 \%$ and icterohaemorrhagiae and grippotyphosa in CE, icterohaemorrhagiae in $P B$ and pyrogenes in SP; horses - 29\% and serovar icterohaemorrhagiae in $P R, S C, S P, R J$ e $M G$, grippotyphosa in $M T$, pyrogenes in $P B$ and patoc in $R S$; swines $24.46 \%$ and serovar grippotyphosa and icterohaemorrhagiae in $M G$, pomona in $R S$, pomona and icterohaemorrhagiae in PE and $R J$, autumnalis in CE and icterohaemorrhagiae in $G O, P R, S C$ e $S P$.

Key words: sorology, agglutination, leptospirosis, buffalo, sheep, goat, horse, swine, dog.

\section{INTRODUÇÃO}

No Brasil, os exames de soroaglutinação microscópica aplicados ao diagnóstico da leptospirose têm registrado a predominância dos sorovares (sv) autumnalis e javanica em ovinos; canicola, icterohaemorrhagiae, panama, autumnalis e celedoni nos caprinos; wolffi, pomona e icterohaemorrhagiae em bubalinos; pomona em suínos; icterohaemorrhagiae e pomona em eqüinos; icterohaemorrhagiae, canicola e pyrogenes nos cães (ABUCHAIN, 1991; ALVES et al., 1996; BRITO, 1985; CALDAS, 1985; CARVALHO et al., 1990; CORDEIRO et al., 1974; FURTADO et al., 1997;.GIORGI et al., 1981a; GIORGI et al., 1981b; GIRIO, 1984; JOUGLARD, 1999; LANGONI et al., 1997; LARSSON et al., 1984; OLIVEIRA, 1977; OLIVEIRA et al., 1987; QUERINO, 1999; RAMOS et al., 1981; SANDOVAL et al., 1979 SANTA ROSA \& CASTRO, 1963; SANTA ROSA et al., 1969/70; VIEGAS et al., 1980; VIEGAS, 1985; YANAGUITA $\boldsymbol{e t}$ al., 1982; YASUDA, 1980).

Os resultados de testes sorológicos aplicados ao diagnóstico da leptospirose dependem da técnica empregada, da coleção de antígenos utilizada, do ponto de corte da reação e também de variáveis relacionadas à localização das propriedades, período do ano em que as colheitas foram efetuadas e da movimentação dos animais (FAINE, 1982). A interferência destes fatores torna necessária a existência de um sistema de vigilância epidemiológica permanente que possibilite o monitoramento da distribuição espacial dos sv de leptospiras presentes nas diferentes regiões de modo a racionalizar as medidas de controle com especial destaque para a imunoprofilaxia.

$\mathrm{O}$ presente trabalho tem por objetivo a apresentação das variantes sorológicas de leptospiras predominantes em testes sorológicos efetuados em ovinos, caprinos, bubalinos, suínos, eqüinos e cães, originários de diversos estados brasileiro e examinados durante os anos de 1984 a 1997.

\section{MATERIAL E MÉTODOS}

\section{Soroaglutinação microscópica (SAM)}

Foi utilizada a técnica de soroaglutinação microscópica (GALTON et al., 1965; COLE et al., 1973), com uma coleção de antígenos vivos que incluiu 24 variantes sorológicas (sv) de leptospiras patogênicas (australis, bratislava, autumnalis, butembo, castellonis, bataviae, canicola, whitcombi, cinoptery, grippotyphosa, hebdomadis, copenhageni, icterohaemorrhagiae, javanica, panama, pomona, pyrogenes, hardjo, wolffi, shermani, tarassovi, sentot) e duas de leptospiras saprófitas (andamana e patoc). A triagem foi efetuada na diluição de 1:100 e, quando houve aglutinação, os soros foram titulados em uma série geométrica de diluições de razão dois. O título foi dado como a recíproca da maior diluição em que houve aglutinação.

A análise dos resultados considerou como mais provável o sv que apresentou o maior título e maior freqüência. Os animais que apresentaram duas ou mais variantes sorológicas com títulos idênticos foram excluídos desta análise, e considerados reatores para a Leptospira spp.

\section{Registros}

As informações analisadas foram retiradas do banco de dados do Laboratório de Zoonoses Bacterianas do Departamento de Medicina Veterinária Preventiva e Saúde Animal da Faculdade de Medicina Veterinária e Zootecnia da Universidade de São Paulo. Foram considerados: data de entrada, número de soros, procedência e resultado até a titulação. As fichas que não estavam com as informações completas foram eliminadas.

\section{Distribuição dos animais examinados}

Foram computados os registros dos exames de 284 ovinos, 879 de bubalinos, 983 cães, 1.941 de caprinos, 2.903 eqüinos e 8.568 suínos, distribuídos percentualmente por estado da seguinte forma: ovinos - SP (100\%); bubalinos - SP (100\%); cães - SP $(80,7 \%)$, PI $(19,0 \%)$, RS $(0,10 \%)$ e SC $(0,10 \%)$; caprinos - PB $(63,7 \%)$, SP $(33,1 \%)$ e CE $(3,2 \%)$; eqüinos - SP $(79,3 \%)$, RS $(9,98 \%)$, MT (3,99\%), PR (2,5\%), MG (1,96\%), PB (1,3\%), SC $(0,62 \%)$, RJ $(0,17 \%)$ e PI $(0,03 \%)$; suínos - SP (61,91\%), MG (24,38\%), SC (5,95\%), PR (3,67\%), GO $(1,12 \%)$, PE $(0,90 \%)$, RJ $(0,88 \%)$, CE $(0,34 \%)$, RS $(0,3 \%)$, SE $(0,2 \%)$ e MA $(0,1 \%)$.

A distribuição temporal dos animais examinados incluiu: para a espécie ovina $-54,5 \%$ 
referentes aos anos 1996 e 97, 33,3\% referentes a 89 e 90 e $12,2 \%$ aos outros anos; espécie bubalina $21,7 \%$ no intervalo de 1984 a 95 e $78,83 \%$ entre 96 e 97; espécie canina - 16,91\% entre 1984 e 92 e $83,09 \%$ entre 1993 e 97; caprinos - 6,97\% entre 1984 e 91 e $93,09 \%$ entre 1992 e 97 , sendo que $49 \%$ das amostras foram referentes ao ano de 1992; eqüinos - 18,1\% no intervalo de 84 a 90 e $81,9 \%$ de 1991 a 97; suínos - 61,16\% referentes aos anos 90, 91,95 e 96.

\section{RESULTADOS}

As tabelas 1 e 2 apresentam os resultados dos exames de soroaglutinação microscópica aplicada à leptospirose segundo a espécie, o estado e as proporções de municípios, e de animais reatores para pelo menos um sv, bem como os respectivos sv mais prováveis.

A observação das proporções de municípios que incluíram pelo menos um animal positivo no teste de SAM aplicada a leptospirose, segundo a espécie animal, revela que houve ausência de cães positivos em Teresina-PI e Porto Alegre-RS, eqüinos em Teresina-PI; suínos em Salgado, BA e Imperatriz-MA, no entanto, nos demais municípios incluídos as proporções de positivos foram sempre superiores a $50 \%$.
As médias de proporções de animais positivos para pelo menos um sv de leptospira, sobre examinados segundo a espécie animal, apresentam valores crescentes na seguinte ordem: ovinos $(0,70 \%)$; caprinos $(5,61 \%)$; suínos $(14,90 \%)$; cães $(22,84 \%)$, eqüinos $(27,94 \%)$ e bubalinos $(43,7 \%)$.

As variantes mais freqüentes por espécie foram: ovinos - icterohaemorrhagiae; caprinos icterohaemorrhagiae e grippotyphosa no $\mathrm{CE}$, icterohaemorrhagiae na PB e pyrogenes em SP; bubalinos - hardjo e pomona; suínos grippotyphosa e icterohaemorrhagiae em MG, pomona no RS, pomona e icterohaemorrhagiae em $\mathrm{PE}$ e RJ, autumnalis no $\mathrm{CE}$ e icterohaemorrhagiae em GO, PR, SC e SP; eqüinos icterohaemorrhagiae no PR, SC, SP, RJ e MG, grippotyphosa no MT, pyrogenes na $\mathrm{PB}$ e patoc no RS; cães - copenhageni e icterohaemorrhagiae no estado de SP e pyrogenes no PI;

\section{DISCUSSÃO}

O número de exames realizados apresenta distribuição variável por estado com maior concentração (65\%) no Estado da Paraíba para os caprinos, (79,3\%) no Estado de São Paulo para os eqüinos e $(89,9 \%)$ nos Estados de Minas Gerais e São Paulo para os suínos. Esta condição

Tabela 1 - Animais das espécies ovina, bubalina, caprina e canina examinados através da técnica de soroaglutinação microscópica aplicada à leptospirose pelo Laboratório de Zoonoses Bacterianas da Faculdade de Medicina Veterinária e Zootecnia da USP, no período de 1984 a 1997, segundo o Estado Federativo da União, a proporção de amostras positivas e as variantes sorológicas mais prováveis. São Paulo, 2001.

\begin{tabular}{|c|c|c|c|c|c|}
\hline \multirow{3}{*}{$\begin{array}{l}\text { ESPÉCIE } \\
\text { OVINOS }\end{array}$} & \multirow{3}{*}{$\begin{array}{c}\text { ESTADO } \\
\text { SP }\end{array}$} & \multirow{3}{*}{$\begin{array}{c}\text { Proporção de } \\
\text { Municípios positivos } \\
\text { No POS./-EX. (1) } \\
3 / 7\end{array}$} & \multicolumn{2}{|c|}{$\begin{array}{l}\text { Proporção de animais reatores para } \\
\text { pelo menos uma variante sorológica. }\end{array}$} & \multirow{2}{*}{$\begin{array}{l}\text { DUAS VARIANTES SOROLÓGICAS } \\
\text { MAIS PROVÁVEIS } \\
(3)\end{array}$} \\
\hline & & & N POS./-EX. (2) & $(\%)$ & \\
\hline & & & $2 / 284$ & $0,7 \%$ & $\begin{array}{l}\text { Icterohaemorrhagiae }(40,0 \%), \quad \text { butembo } \\
(20,0 \%) \text {, castellonis }(20,0 \%) \text {, hebdomadis } \\
(20,0 \%)^{*}\end{array}$ \\
\hline BUBALINOS & SP & $6 / 7$ & $381 / 879$ & $43,7 \%$ & Hardjo $(43,3 \%)$, wolffi $(32,5 \%)$ \\
\hline \multirow[t]{4}{*}{ CANINOS } & SP & $14 / 25$ & $137 / 795$ & $17,9 \%$ & $\begin{array}{l}\text { Copenhageni }(24,0 \%) \text {, icterohaemorrhagiae } \\
(10,9 \%)\end{array}$ \\
\hline & PI & $1 / 1$ & $37 / 187$ & $19,7 \%$ & Pyrogenes $(100 \%)$ \\
\hline & PR & $0 / 1$ & $0 / 1$ & $0 \%$ & $\ldots$ \\
\hline & RS & $0 / 1$ & $0 / 1$ & $0 \%$ & $\ldots$ \\
\hline \multirow[t]{3}{*}{ CAPRINOS } & SP & $6 / 13$ & $15 / 616$ & $2,4 \%$ & $\begin{array}{l}\text { Pyrogenes }(21,0 \%) \text {, castellonis }(15,7 \%) \text {, patoc } \\
(15,7 \%)^{*}\end{array}$ \\
\hline & $\mathrm{CE}$ & $1 / 1$ & $1 / 63$ & $1,5 \%$ & Icterohaemorrhagiae (100\%) \\
\hline & PB & $3 / 3$ & $65 / 1.262$ & $5,1 \%$ & Icterohaemorrhagiae $(25,0 \%)$, panama $(15,9 \%)$ \\
\hline
\end{tabular}

(1): número de municípios com pelo menos um animal reator sobre o número de municípios examinados;

(2): número de animais positivos sobre o número de animais examinados;

(3): percentual calculado do total de reações aproveitadas uma vez que, nesta análise, aquelas em que houve empate, título mais alto idêntico para dois ou mais variante sorológicas, foram desconsideradas.

...:dados inexistentes

*CITADAS MAIS DE DUAS DEVIDO AO EMPATE NA PERCENTAGEM 
Tabela 2 - Animais das espécies eqüina e suína examinados através da técnica de soroaglutinação microscópica aplicada à leptospirose pelo Laboratório de Zoonoses Bacterianas da Faculdade de Medicina Veterinária e Zootecnia da USP, no período de 1984 a 1997, segundo o Estado Federativo da União, a proporção de amostras positivas e as variantes sorológicas mais prováveis. São Paulo, 2001.

\begin{tabular}{|c|c|c|c|c|c|}
\hline \multirow[t]{2}{*}{ ESPÉCIE } & \multirow[t]{2}{*}{ ESTADO } & \multirow{2}{*}{$\begin{array}{c}\text { Proporção de } \\
\text { Municípios positivos } \\
\mathrm{N}^{\circ} \text { POS./-EX (1) }\end{array}$} & \multicolumn{2}{|c|}{$\begin{array}{l}\text { Proporção de animais reatores para } \\
\text { pelo menos uma variante sorológica. }\end{array}$} & \multirow{2}{*}{$\begin{array}{c}\text { DUAS VARIANTES SOROLÓGICAS } \\
\text { MAIS PROVÁVEIS } \\
(3)\end{array}$} \\
\hline & & & $\mathrm{N}^{\circ}$ POS./-EX. (2) & $(\%)$ & \\
\hline \multirow{9}{*}{ EQUINOS } & PI & $0 / 1$ & $0 / 1$ & $0,0 \%$ & $\ldots$ \\
\hline & PB & $1 / 1$ & $5 / 39$ & $12,8 \%$ & Pyrogenes $(47,8 \%)$, patoc $(45,0 \%)$ \\
\hline & RS & $2 / 2$ & $51 / 290$ & $17,5 \%$ & Patoc $(47,7 \%)$, pyrogenes $(25,0 \%)$ \\
\hline & PR & $2 / 2$ & $13 / 74$ & $17,5 \%$ & Icterohaemorrhagiae (100\%) \\
\hline & $\mathrm{SC}$ & $1 / 1$ & $5 / 18$ & $27,7 \%$ & $\begin{array}{l}\text { Icterohaemorrhagiae }(62,5 \%) \text {, } \\
\text { castellonis+autumnalis }(12,5 \%)\end{array}$ \\
\hline & SP & $60 / 72$ & $686 / 2.303$ & $29,7 \%$ & $\begin{array}{l}\text { Icterohaemorrhagiae }(29,5 \%), \quad \text { pyrogenes } \\
(7,27 \%)\end{array}$ \\
\hline & MT & $3 / 3$ & $42 / 116$ & $36,2 \%$ & Grippotyphosa $(66,6 \%)$, patoc $(33,4 \%)$ \\
\hline & RJ & $1 / 1$ & $2 / 5$ & $40,0 \%$ & Icterohaemorrhagiae $(100 \%)$ \\
\hline & MG & $4 / 4$ & $40 / 57$ & $70,1 \%$ & Icterohaemorrhagiae $(25,0 \%)$, hardjo $(12,0 \%)$ \\
\hline \multirow{11}{*}{ SUÍNOS } & BA & $0 / 1$ & $0 / 78$ & $0,0 \%$ & $\ldots$ \\
\hline & MA & $0 / 1$ & $0 / 10$ & $0,0 \%$ & $\ldots$ \\
\hline & MG & $8 / 8$ & $1.334 / 2.092$ & $6,4 \%$ & $\begin{array}{l}\text { Grippotyphosa }(33,3 \%) \text {, icterohaemorrhagiae } \\
(18,8 \%)\end{array}$ \\
\hline & RJ & $3 / 3$ & $6 / 76$ & $7,8 \%$ & Pomona $(33,3 \%)$, icterohaemorrhagiae $(33,3 \%)$ \\
\hline & PR & $7 / 7$ & $28 / 315$ & $8,8 \%$ & $\begin{array}{l}\text { Icterohaemorrhagiae }(66,6 \%) \text {, grippotyphosa } \\
(11,1 \%)\end{array}$ \\
\hline & $\mathrm{GO}$ & $1 / 2$ & $9 / 96$ & $9,3 \%$ & $\begin{array}{l}\text { Icterohaemorrhagaie }(66,6 \%) \text {, grypotyphosa+ } \\
\text { icterohaemorrhagiae+ pyrogenes }(33,4 \%)\end{array}$ \\
\hline & $\mathrm{SC}$ & $1 / 2$ & $52 / 511$ & $10,1 \%$ & $\begin{array}{l}\text { Icterohaemorrhagiae }(62,5 \%), \text { autumnalis } \\
(12,5 \%)\end{array}$ \\
\hline & SP & $60 / 74$ & $639 / 5.311$ & $12,0 \%$ & $\begin{array}{l}\text { Icterohaemorrhagiae }(44,8 \%), \text { autumnalis } \\
(7,8 \%)\end{array}$ \\
\hline & $\mathrm{CE}$ & $1 / 1$ & $8 / 30$ & $26,6 \%$ & $\begin{array}{l}\text { Autumnalis } \quad(50,0 \%), \text { icterohaemorrhagiae } \\
(50,0 \%)\end{array}$ \\
\hline & RS & $1 / 2$ & $11 / 29$ & $37,9 \%$ & Pomona $(100 \%)$ \\
\hline & $\mathrm{PE}$ & $3 / 3$ & $9 / 20$ & $45,0 \%$ & Pomona $(47,7 \%)$, icterohaemorrhagiae $(47 \%)$ \\
\hline
\end{tabular}

(1): número de municípios com pelo menos um animal reator sobre o número de municípios examinados;

(2): número de animais positivos sobre o número de animais examinados;

(3): percentual calculado do total de reações aproveitadas uma vez que, nesta análise, aquelas em que houve empate, título mais alto idêntico para dois ou mais variante sorológicas, foram desconsideradas.

...:dados inexistentes

*CITADAS MAIS DE DUAS DEVIDO AO EMPATE NA PERCENTAGEM

impossibilita a análise comparativa da freqüência de reatores e por estado, no entanto não invalida a contribuição dos resultados obtidos particularmente devido ao número de sorovares de leptospiras incluídos na coleção de antígenos empregada

Como a demanda do diagnóstico laboratorial da leptospirose está usualmente associada à suspeita clínica e, no presente experimento, não houve um planejamento para que os animais examinados fossem representativos das populações dos estados e municípios trabalhados, os resultados obtidos não podem ser interpretados como indicadores de prevalência. No entanto contribuem para o conhecimento da distribuição dos sorogrupos grassantes nas espécies examinadas, regiões e período estudado.
A despeito de ter sido observada a ausência de eqüinos reatores para a leptospirose nos materiais originários de Teresina (PI) e em 12 municípios de SP; cães reatores nos materiais originários de Londrina (PR), Porto Alegre (RS) e de 11 municípios de $\mathrm{SP}$; de suínos nos materiais originários de Braço do Norte (SC), Porto Alegre (RS), Salgado (BA), Goiás (GO) e de Imperatriz, $\mathrm{MA}$, além de 14 municípios de SP; caprinos nos materiais de sete municípios de SP; ovinos de quatro municípios de SP e bubalinos de Nova Odessa (SP) em todos os outros 193 municípios que enviaram materiais houve pelo menos um indivíduo da respectiva espécie animal com resultado positivo para a leptospirose o que demonstra a ampla difusão da infecção nas populações animais de diversos estados do território nacional. 
Nos ovinos, a variante icterohaemorrhagiae predominou nos animais do Estado de São Paulo, discordando dos achados de SANTA ROSA \& CASTRO (1963) em SP; SANTA ROSA et al. (1969/70) e VIEGAS et al. (1980) que encontraram a predominância do SV autumnalis e de VIEGAS (1985) que verificou o sv javanica. A despeito dos resultados de VIEGAS et al. (1980) e VIEGAS (1985) terem sido obtidos em animais do estado da Bahia que pode apresentar uma distribuição regional distinta do Estado de São Paulo, o sv encontrado no presente estudo icterohaemorrhagiae apresenta uma associação universal com roedores sinantrópicos (FAINE, 1982) e sugere que, na atualidade, este tipo de reservatório deva estar envolvido com as criações de ovinos.

Nos búfalos, o predomínio dos SV hardjo e wolffi discorda dos achados de SANTA ROSA et al. (1969/70), GIRIO (1984) e LANGONI et al. (1997) que encontraram maior freqüência de reatores para o sv wolffi e de SANDOVAL et al. (1979) e GIORGI et al. (1981b) com o sv pomona, destaque-se contudo que o sv hardjo só foi incluído na coleção de antígenos empregada por LANGONI et al. (1997) e o mesmo não aplicou a sistemática de análise de coaglutinações adotada no presente estudo.

$\mathrm{Na}$ espécie canina, o resultado encontrado em SP, com predomínio do sv copenhageni diferiu do dos achados de SANTA ROSA et al. (1969/70), YASUDA (1980), GIORGI et al. (1981b), FURTADO et al. (1997) e JOUGLARD (1999) e QUERINO (1999). Ressalte-se, todavia, que na atualidade o sv copenhageni tem sido o mais freqüentemente isolado em casos de leptospirose em seres humanos no Estado de São Paulo (SAKATA et al., 1992) o que sugere a participação dos roedores sinantrópicos como fonte comum tanto para humanos como para os cães e indica a revisão dos sorovares empregados na formulação de bacterinas destinadas aos cães que usualmente são produzidas com os SV icterohaemorrhagiae e canicola e não incluem o copenhageni.

Nos caprinos, o predomínio do SV icterohaemorrhagiae nos Estados do CE e PB concorda com SANTA ROSA \& CASTRO (1963) e ALVES et al. (1996) e discorda de SANTA ROSA et al. (1969/70), CALDAS (1985), BRITO (1985) e VIEGAS (1985) que constaram predomínio dos sv canicola, autumnalis e celledoni. A variante pyrogenes identificada como a mais freqüente em SP ainda não havia sido referida em outras investigações nesta espécie animal e suscita a realização de novos estudos com isolamento e tipificação do agente para a real confirmação do sv implicado, pois o teste de SAM é uma prova sorogrupo específica (FAINE, 1982).

Nos eqüinos, o predomínio da variante icterohaemorrhagiae concorda com os resultados obtidos por GIORGI et al. (1981b), YANAGUITA et al. (1982) e ABUCHAIN (1991) e discorda de SANTA ROSA et al. (1969/70); GIORGI et al. (1981a) e CORDEIRO et al. (1974) que encontraram a predominância da variante pomona. A presença das variantes: pyrogenes na $\mathrm{PB}$, patoc no RS e grippotyphosa no MT como as mais prováveis ainda não havia sido observada no Brasil. Reações para os Sv. icterohaemorrhagiae e pomona sugerem respectivamente a participação de roedores sinantrópicos e de suínos, tradicionalmente conhecidos como hospedeiros de manutenção destes sorovares (FAINE, 1982), no entanto as outras associações sugerem a realização de novas investigações a fim de ser identificada a origem dos focos.

$\mathrm{Na}$ espécie suína, o predomínio do sorovar pomona foi observado nos estados do RJ, RS e PE comprovando os estudos de SANTA ROSA et al. (1969/70), OLIVEIRA (1977), RAMOS et al. (1981), VIEGAS et al. (1980), LARSSON et al. (1984) e OLIVEIRA et al. (1987); no entanto os resultados obtidos no $\mathrm{PR}, \mathrm{GO}$ e $\mathrm{SC}$ que acusaram o sv. icterohaemorrhagiae como mais freqüente reafirmam os achados de CARVALHO et al. (1990) e VIEGAS et al. (1980) e sugerem que a evolução da suinocultura nacional, observada nos últimos anos deve ter modificado o perfil da infecção por leptospiras, na qual o sv pomona tradicionalmente mantido pelos próprios suínos, está sendo substituído pelo icterohaemorragiae cujo achado fomenta a implantação de procedimentos destinados ao controle de roedores sinantrópicos como parte do manejo das criações.

Os resultados observados no presente estudo e as discrepâncias encontradas com os achados de outros investigadores podem ser atribuídas a fatores metodológicos como ponto de corte, modalidade de teste e coleção de antígenos empregada, no entanto, também não pode ser excluída a hipótese da expansão da disseminação de um determinado sorovar na dependência de fatores ambientais ligados ao manejo e a movimentação de animais (FAINE, 1982).

A despeito de, no presente estudo, ter sido introduzida a interpretação dos resultados com caracterização dos sorovares com maior titulação e eliminação das reações que apresentaram títulos empatados, os resultados conclusivos só poderão ser obtidos com investigações que incluam o isolamento e a tipificação das leptospiras encontradas. 


\section{CONCLUSÕES}

Houve diferença nas variantes sorológicas de leptospirose mais prováveis segundo a espécie animal e o estado onde vivem os referidos animais. As variantes mais freqüentes por espécie foram: ovinos: icterohaemorrhagiae; bubalinos: hardjo seguida de wolffi; cães: copenhageni e icterohaemorrhagiae no estado de SP e pyrogenes no PI; caprinos: icterohaemorrhagiae no $\mathrm{CE}$, icterohaemorrhagiae e panama na $\mathrm{PB}$ e pyrogenes em SP; eqüinos: icterohaemorrhagiae no $\mathrm{MG}$, $\mathrm{PR}$, RJ, SC e SP, grippotyphosa e patoc no MT, pyrogenes e patoc na PB e patoc e pyrogenes no RS; suínos: grippotyphosa seguida de icterohaemorrhagiae em MG, pomona no RS, pomona e icterohaemorrhagiae em PE e RJ, autumnalis $e$ icterohaemorrhagiae no $C E$ e icterohaemorrhagiae em GO, PR, SC e SP.

\section{AGRADECIMENTOS}

Trabalho patrocinado pela FAPESP, Processo n.97/02882-6.

\section{REFERÊNCIAS BIBLIOGRÁFICAS}

ABUCHAIN, D.M. Presença de aglutininas antileptospira em soro de eqüinos no Estado do Rio Grande do Sul. Arquivos da Faculdade de Veterinária UFRGS, Porto Alegre, v.19, p.9-14, 1991

ALVES, C.J., VASCONCELlOS, S.A., CAMARGO, C.R.A., et al. Influência dos fatores ambientais sobre a proporção de caprinos soro-reatores para a leptospirose em cinco centros de criação do Estado da Paraíba, Brasil. Arquivos do Instituto Biológico de São Paulo, v.63, n.2, p.11-18, 1996.

BRITO, D.E.M.W. Aspectos zoo-sanitários em caprinos de diferentes formas de exploração no sul de Goiás e Distrito Federal. Belo Horizonte, 1985. 65p. Dissertação (Mestrado) - Escola de Medicina Veterinária, Universidade Federal de Minas Gerais.

CALDAS, E.M. Investigação comparativa de estirpes apatogênicas para o diagnóstico sorológico da leptospirose animal. Salvador, 1985. 37p. Tese (Concurso para Prof Titular) - Escola de Medicina Veterinária, Universidade Federal da Bahia, 1985.

CARVALHO, L.F.O.S., CARVALHO, M.B., GIRIO, R.J.S., et al. Investigação sorológica de fêmeas suínas descartadas para abate por transtornos reprodutivos diversos. Ciência Veterinária, v.4, n.2, p.6-8, 1990.

COLE, JR., SULZER, C.R., PULSSELY, P.R. Improved microtechinique for the leptospiral microscopic aglutination. Applied Microbiology, v.25, n.6, p.976-980, 1973.

CORDEIRO, F., RAMOS, A.A., BATISTA JR., J.A. Aglutininas antileptospira em soros de eqüinos de Minas Gerais. Pesquisa Agropecuária Brasileira Veterinária, v.9, p.45-48, 1974.

FAINE, S. (ed). Guidelines for the control of leptospirosis. Geneva : World Health Organization, 1982. 171p. (WHO off set Publication, 67)
FURTADO, L.R.I., AVILA, M.O., FEHLBERG, M.F.B., $\boldsymbol{e t}$ al. Prevalência e avaliação de fatores de risco à leptospirose canina, no município de Pelotas-RS. Arquivos do Instituto Biológico, São Paulo, v.64, n.1, p.57-61, 1997.

GALTON, M.M., SULZER, C.R., SANTA ROSA, C.A., et al. Application of a microtechnique to the agglutination test for leptospiral antibodies. Applied Microbiology, v.13, n.1, p.81-85, 1965

GIORGI, W., TERUYA, J.M., MACRUZ, R., et al. Leptospirose em eqüinos: inquérito sorológico e isolamento de Leptospira icterohaemorrhagiae de feto abortado. Revista do Instituto Biológico, v.47, n.2, p.47-53, 1981a.

GIORGI, W. TERUYA, J.M.; SILVA, A.S. Leptospirose: Resultados das soroaglutinações realizadas no Instituto Biológico de São Paulo durante os anos de 1974/1980. Biológico, v.47, n.11, p.299-309, 1981b.

GIRIO, R.J.S. Estudo comparativo de quatro estirpes apatogênicas utilizadas em teste de triagem para o diagnóstico sorológico da leptospirose em búfalos (Bubalus bubalis). São Paulo, 1984. 52p. Dissertação (Mestrado) - Instituto de Ciências Biomédicas, Universidade de São Paulo, 1984.

JOUGLARD, S.D.D. Prevalência da leptospirose canina, fatores de risco e constituição da população no meio rural do Município de Pelotas, RS. Pelotas, 1999. 73p. Dissertação (Mestrado) - Faculdade de Medicina Veterinária, Universidade Federal de Pelotas, 1999.

LANGONI, H., DEL FAVA, C., ABRAL, K.G., et al. Epidemiological survey on anti-leptospire aglutinins in buffaloes from Vale do Ribeira, SP., State (Brazil). In: WORLD BUFFALO CONGRESS, 5, 1997, Caserta, Italy, p.622-625.

LARSSON, C.E., YASUDA, P.H., SANTA ROSA, C.A. Leptospirose suína. Inquérito sorológico e bacteriológico em municípios dos Estados de São Paulo, do Paraná e de Santa Catarina. Revista da Faculdade de Medicina Veterinária e Zootecnia da Universidade de São Paulo, v.21, n.1, p.43$50,1984$.

OLIVEIRA, S.J. Presença de aglutininas antileptospiras em suínos e bovinos, com e sem sinais de infecção, no Rio Grande do Sul. Bol IPVDF, v.4, p.57-64, 1977.

OLIVEIRA, S.J., GUIZZARD, I.I., VIDOR, T., et al. Testes sorológicos para diagnóstico de leptospirose, peste suína africana e doença de Aujeszky em granjas de reprodutores suínos no Rio Grande do Sul (ano 1994). Arquivos Brasileiros de Medicina Veterinária e Zootecnia, v.39, n.3, p.451-460, 1987.

QUERINO, A.M.V. Estudo dos fatores de risco associados à leptospirose em cães do Município de Londrina - Paraná. Londrina, 1999. 34p. Dissertação (Mestrado) - Faculdade de Medicina Veterinária e Zootecnia da Universidade Estadual de Londrina, 1999.

RAMOS, A.A., CORDEIRO, F., GUIDA, H.G. Inquérito sorológico de leptospirose em suínos do Estado do Rio de Janeiro e região limítrofe. Pesquisa Veterinária Brasileira, v.1, n.3, p.81-83, 1981.

SAKATA, E.E., YASUDA, P.H., ROMERO, E.C., et al. Sorovares de Leptospira interrogans isolados de casos de leptospirose humana em São Paulo, Brasil. Revista do Instituto de Medicina Tropical, v.34, p.217-221, 1992. 
SANDOVAL, L.A., ARRUDA, N.M., TERUYA, J.M., $\boldsymbol{e}$ t al. Pesquisas em bubalinos: prevalência da brucelose e leptospirose no Estado de São Paulo, Brasil. Arquivos do Instituto Biológico, v.45, n.11-12, p.209-212, 1979.

SANTA ROSA, C.A., CASTRO, A.F.P. Presença de aglutininas anti-leptospiras em soros de ovinos e caprinos no Estado de São Paulo. Arquivos do Instituto Biológico de São Paulo, v.30, p.93-98, 1963.

SANTA ROSA, C.A., CASTRO, A.F.P., SILVA, A.S. Nove anos de leptospirose no Instituto Biológico de São Paulo. Revista do Instituto Adolfo Lutz, v.29/30, p.19-27, 1969/1970.

VIEGAS, E.A. Estudo de novos sorotipos de leptospiras apatogênicas na prova de soroaglutinação microscópica para o diagnóstico da leptospirose caprina e ovina. São
Paulo, 1985. 63p. Dissertação (Mestrado) - Instituto de Ciências Biomédicas, Universidade de São Paulo, 1985.

VIEGAS, E.A., VIEGAS, S.A.R.A., CALDAS, E.M. Aglutininas anti-leptospira em hemo-soro de caprinos e ovinos no Estado da Bahia. Arquivo da Escola de Medicina Veterinária da Universidade Federal da Bahia, v.5, n.1, p.20-34, 1980.

YANAGUITA, R.M., SANTA ROSA, C.A., ROSA, R.R. Prevalência de aglutininas anti-leptospiras em eqüinos mantidos para produção de soros terapêuticos. Revista Microbiológica. v.13, n.1, p.22-25, 1982.

YASUDA, P.H.; SANTA ROSA C.A.; MYERS, D.M. The isolation of leptospires from stray dogs in the city of Sâo Paulo, Brazil. International Journal of Zoonosis, v.7, p.131-134, 1980. 